\title{
Comparison between Two-Layer Density Gradient and Three-Layer Density Gradient Technique for Sperm Preparation at Aster Fertility Clinic, Dr. Hasan Sadikin General Hospital
}

\author{
Nanang Winarto Astarto, ${ }^{1}$ Dian Tjahyadi, ${ }^{1}$ Sintya Jatnikasari ${ }^{2}$ \\ ${ }^{1}$ Department of Obstetrics and Gynaecology, Faculty of Medicine, Universitas Padjadjaran-Dr. Hasan Sadikin \\ General Hospital \\ ${ }^{2}$ Aster Fertility Clinic, Dr. Hasan Sadikin General Hospital
}

\begin{abstract}
Objective: To compare the efficacy of sperm preparation techniques modified by two layer density gradient technique $(80 \%, 40 \%)$.

Methods: Sperm preparation was performed by two- and three-layer density gradient technique by centrifugation at 500 rpm for 20 minutes using SpermGrad (Vitrolife, Sweden).

Results: The average concentration of total sperm preparation by two-layer density gradient was 23.19 million with a standard deviation of 11.9 million while the result of three-layer density gradient preparation obtained a mean total sperm concentration of 19.64 million with a standard deviation of 10.033 million. The mean total concentration of sperm in three-layer was lower than two-layer density gradient with $\mathrm{p}<0.001$.
\end{abstract}

Received:

March 21, 2014

Conclusions: This study concludes that there was a significant difference between the concentration of sperm prepared using two-layer and three-layers density

Revised:

May 12, 2014 gradient technique.

Accepted:

June 24, 2014

Keywords: Density gradient, spermatozoa

IJIHS. 2014;2(1):40-4

\section{Introduction}

Current assisted reproductive technologies such as intrauterine insemination (IUI) and in vitro fertilization (IVF)/intracytoplasmic sperm injection (ICSI) have been widely applied in the treatment of infertility. Ejaculated spermatozoa should be separated from seminal plasma in order to function properly. The presence of prostaglandins in seminal plasma may cause uterine contractions. In addition, seminal plasma also contains several substances that may reduce the ability of sperm to fertilize. Therefore, sperm preparation techniques have become an integral part in assisted reproductive techniques (ART). ${ }^{1}$

Various kinds of sperm preparation techniques have been developed, such as swim-up, swimdown, and density gradient techniques. These

\section{Correspondence:}

Nanang Winarto Astarto, Department of Obstetrics and Gynaecology, Faculty of Medicine, Universitas PadjdajaranDr. Hasan Sadikin General Hospital

Jl. Pasteur No. 38, Bandung, Indonesia

e-mail:nw_astarto@yahoo.com techniques aimed to produce a ready to use sperm suspensions that are free from plasma, non-motile sperm, debris, leukocytes, and other contaminants, with a high recovery of motile sperms. Sperm separation by density gradient has become a standard technique in many ART laboratories because it can be done easily and quickly resulting a high motile sperm recovery. In addition, the density gradient has become a common procedure that is widely known. ${ }^{2}$

Percoll density gradient procedure has been used extensively in a variety of ART procedures. However, several studies reported that the Percoll gradient containing endotoxin can cause irritation to the tissue after IUI and the silica particles might damage the sperm membrane. ${ }^{2}$ Consequently, a wide range of products and density gradient procedures were developed and have produced different effects on the quantity of sperm preparation outcome and fertilization rate. $^{3}$

Aster Clinical Laboratory, Dr. Hasan Sadikin General Hospital, routinely uses three-layer density gradient technique $(90 \%, 70 \%, 40 \%)$ by 
centrifugation at $500 \mathrm{rpm}$ for 20 minutes using SpermGrad $^{\circledR}$ (Vitrolife, Sweden) while several journals and many studies reported the use of two-layer density gradient technique with 1,750 rpm centrifugation for $10 \mathrm{~min}^{4}$ The purpose of this study was to determine which density gradient technique is more effective and efficient between the three-layer density gradient $190 \%$, $70 \%, 40 \%)$ and two-layer density gradient $(80 \%$, $40 \%)$.

\section{Methods}

Objects

The sperm samples were obtained from 25 adults who signed up for routine sperm analysis in Aster Clinical Laboratory Dr. Hasan Sadikin General Hospital, Bandung. Each semen sample was divided into two equal parts and each aliquot was prepared using three-layer and two-layer density gradient density techniques. The research was conducted in the period of September and October 2010. The research took place in Aster IVF Clinic, Dr. Hasan Sadikin General Hospital.

Semen Samples and Medium

Semen samples were collected through masturbation and collected in sterile containers after 3 days of sexual abstinence. Each sample was examined for volume, motility, and sperm concentration using the World Health Organization (WHO) 1999 manual standard. Every post liquefaction sperm samples were divided into two equal parts and each aliquot was prepared using three-layers discontinuous density gradient $(90 \%, 70 \%, 40 \%)$ with a speed of $500 \mathrm{rpm}$ centrifugation for 20 minutes, followed by two-layer discontinuous density gradient $(80 \%, 40 \%)$ with a speed of $1,750 \mathrm{rpm}$ for 10 minutes.

SpermRinse $^{\circledR}$ medium (Vitrolife, Sweden) were equilibrated for 24 hours in a $5 \% \mathrm{CO}_{2}$ incubator at $37^{\circ} \mathrm{C}$. Gradient stock solution $100 \%$ SpermGrad ${ }^{\circledR}$ (Vitrolife, Sweden) was diluted using SpermRinse ${ }^{\circledR}$ to generate a concentration gradient medium, respectively $90 \%, 80 \%, 70 \%$, and $40 \%$.

\section{Sperm Preparation}

Experiment 1: Sperm preparation using three layers density gradient (90\%, 70\%, 40\%)

A total of $1 \mathrm{~mL}$ aliquot SpermGrad ${ }^{\circledR} 40 \%$ was inserted into the tube. SpermGrad ${ }^{\circledR} 70 \%$ and $90 \%$ were placed respectively at the bottom of the tube. About $1 \mathrm{~mL}$ of semen was inserted into the tube diligently through the tube wall. All samples were then centrifuged at 500 rpm for 20 minutes. After centrifugation, $1 \mathrm{~mL}$ SpermGrad $40 \%$ and $70 \%$ and $0.5 \mathrm{~mL}$ of $90 \%$ SpermGrad were discarded. Residual $0.5 \mathrm{~mL}$ SpermGrad 90\% was diluted in $2 \mathrm{~mL}$ medium and centrifuged at $1,500 \mathrm{rpm}$ for 10 minutes. Sperm pellet was then diluted in $0.5 \mathrm{~mL}$ SpermRinse ${ }^{\circledR}$.

Experiment 2: Separation of sperm density gradient using two layers of $80 \%$ and $40 \%$

Approximately $1 \mathrm{~mL}$ aliquot SpermGrad ${ }^{\circledR}$ $40 \%$ was put into the tube, followed by $1 \mathrm{~mL}$ SpermGrad ${ }^{\circledR} 80 \%$ at the bottom of the tube. One $\mathrm{ml}$ aliquot of semen was inserted into the tube

Table 1 Sperm Concentration Differences between Three-Layer and Two-Layer Density Gradient Techniques

\begin{tabular}{lccc}
\hline \multirow{2}{*}{ Concentration } & $\begin{array}{c}\text { Three-Layer Density Gradient } \\
(\mathbf{n}=\mathbf{2 5}) \text { (in milllions) }\end{array}$ & $\begin{array}{c}\text { Two-Layer Density Gradient } \\
(\mathbf{n = 2 5}) \text { (in milllions) }\end{array}$ & p Value \\
\hline Mean (SD) & $21.96(11.01)$ & $27.32(13.27)$ & 0.0001 \\
Range & $12-63$ & $7-49$ & \\
SD: standard deviation; The p-value is calculated based on the Independent t-test
\end{tabular}

Table 2 Sperm Motility Differences between Three-Layer and Two-Layer Density Gradient Techniques

\begin{tabular}{lccc}
\hline \multicolumn{1}{c}{ Motility } & $\begin{array}{c}\text { Three-Layer Density Gradient } \\
(\mathbf{n = 2 5}) \text { (in percent) }\end{array}$ & $\begin{array}{c}\text { Two-Layer Density Gradient } \\
(\mathbf{n = 2 5} \text { (in percent) }\end{array}$ & p Value \\
\hline Mean (SD) & $89.24(7.16)$ & $84.32(8.89)$ & 0.0001 \\
Range & $80-95$ & $70-90$ & \\
\hline
\end{tabular}

Description: The p-value calculated by Wilcoxon signed-rank test 
diligently. The sample was centrifuged at 1,750 rpm for 10 minutes. The pellet that was obtained using $2 \mathrm{~mL}$ SpermRinse ${ }^{\circledR}$ was washed, rotated at 1,500 rpm for 5 minutes and the pellet was then diluted in $0.5 \mathrm{~mL}$ SpermRinse ${ }^{\circledR}$.

Measured parameters of this study are the value of recovery of motile sperm, motility percentage, sperm concentration, and total concentration of the post washing sperms.

\section{Results}

The mean concentration of sperm preparation technique with two-layer density gradient was 27.32 millions with a standard deviation of 13.272 millions. For the three-layers density gradient preparation, the mean sperm concentration obtained was 21.96 millions with a standard deviation of 11.013 millions. A visible difference between the average value of two-layer density gradient and three-layer density gradient was 5.36 with a standard deviation of 5.384 with a $p$ value of $<0.001$. It can be concluded that there were significant differences between the concentration of sperm preparation prepared by density gradient techniques using two-layer and three-layer density gradient.

The average concentration of the sperm preparation obtained by three-layer gradient technique was 21.96 millions with a standard deviation of 11.013 millions. The mean concentration of sperm preparation by two layer density gradient was 27.32 millions with a standard deviation of 13.272 millions. The visible difference between the average value of two-layer and three-layer density gradient was 5.36 millions with a standard deviation of 5.384 millions. There was a significant difference between the concentration of sperms in two-layer and three-layer density gradient preparations, statistically.

The mean sperm motility that was obtained with three-layer density gradient technique was 89.24 percent with a standard deviation of 7,155 percent. The mean sperm motility preparation by two-layer density gradient group was 84.32 percent with a standard deviation of 8.892 percents. The difference between the average value of two-layer and three-layer density gradient motility density was 4.92 with a standard deviation of 5.291. The statistical test result showed that there was a significant difference of sperm motility between the two-layer and three-layer density gradient preparation.

The mean total sperm concentration in three-layer density gradient technique group was 19.64 millions with a standard deviation of 10.033 millions. The mean concentration of total sperm preparation with two-layers density gradient was 23.19 millions with a standard deviation of 11.902 millions. The difference between the average value of two-layers and three-layers density gradient was 3.54 millions with a standard deviation of 4.390 millions. There was a significant difference between the concentration of sperms in two-layer and threelayer density gradient preparations.

\section{Discussion}

The purpose of sperm preparation in assisted reproductive technology is to obtain recovery of sperm from the seminal fluid with the highest results and without any iatrogenic effects that can reduce sperm motility, viability, and capability to fertilize. Discontinuous density gradient appears to be the most effective method to remove seminal fluid and other cellular elements in the semen. ${ }^{5}$ Sperm separation technique with a density gradient can vary, by using one layer up to ten layers. However, the most widely known technique uses two or three layers. Many studies reported that the result of the sperm preparation using four-layer density gradient has lower quality than the two or three layers technique. ${ }^{6}$

This study demonstrated that the motility of sperm prepared using three-layer density gradient technique was higher than the twolayer density gradient technique. It was caused

Table 3 Distribution of Total Sperm Concentrations between Three-Layer and Two-Layer Density Gradient Techniques

\begin{tabular}{lccc}
\hline \multicolumn{1}{c}{ Motility } & $\begin{array}{c}\text { Three-Layer Density Gradient } \\
(\mathbf{n}=\mathbf{2 5}) \text { (in millions) }\end{array}$ & $\begin{array}{c}\text { Two-Layer Density Gradient } \\
(\mathbf{n}=\mathbf{2 5}) \text { (in millions) }\end{array}$ & p Value*) \\
\hline Mean (SD) & $19.64(10.03)$ & $23.19(11.9)$ & 0.0001 \\
Range & $6.30-44.10$ & $9.10-53.55$ & \\
\hline
\end{tabular}

SD: standard deviation; The $\mathrm{p}$-value calculated by independent t-test 
by the number of Percoll layers in the three-layer technique that was higher than in the two-layer density gradient technique. This resulted in only sperms with excellent motility were able to pass through the three-layers density gradient of percoll. Our results are relevant with Chen's study that suggested PureSperm 3-layer gradients for sperm preparation have been reported to result in specimens with highly motile and morphologically normal spermatozoa. ${ }^{2}$

Total concentration of sperm in the two-layer density gradient technique was higher than in the three-layer density gradient technique group. This suggests that the two-layer density gradient technique produces a higher sperm recovery technique of density gradient than the threelayer technique. The number of layers in the three-layer density gradient percoll technique was higher than in the two-layer density gradient technique; therefore the ability to select sperm in the three-layer density gradient technique is higher, with only a small amount of sperms was able to pass through. Hence, the concentration of sperms in the three-layer density gradient technique was lower than that of the two-layer density gradient technique group.

The two-layer density gradient is a suitable method for sperm preparation in IUI that requires a high number of sperms to reach a higher chance of successful fertilization. The concentration that resulted from this method is higher than from the three-layer method. Although the recovery motility is lower than that of the three-layer technique, it is still considered as normal.

The swim-up technique and the density gradient have different efficiency in separating the sperms. The sperms isolated with the technique are clean and motile, but damaged by the reactive oxygen species (ROS) with high deoxyribonucleic acid (DNA) integrity. The sperms isolated with the density gradient centrifugation are not damaged by the ROS but with low DNA integrity. ${ }^{7}$ Another study showed that the sperms which are obtained through twolayer density gradient centrifugation provide spermatozoa with a higher quality in terms of the motility, viability, and low DNA fragmented as compared to those which are obtained by the other conventional sperm preparation methods. ${ }^{8}$

A comparative study of two-layer density gradient technique and swim-up method showed that the outcome of two-layer density technique is better than the swim-up method. Two-layer density gradient method is superior to the swim-up method for sperm preparation and also more rapid and simpler technique compared to the swim-up method. Spermatozoa separated in two-layer gradient yields a higher number of motile sperms that can withstand cryopreservation that changes better than the specimens prepared by swim-up method. It may provide higher rates of recovery of mature, motile sperms in the specimens processed for ART, which may result in higher fertilization and pregnancy rates. ${ }^{9,10}$

The three-layer density gradient is a more suitable method for IVF and ICSI techniques. The three-layer density has a higher recovery motility value than the two-layers, despite its lower concentration. Since IVF techniques require a higher motility without considering the concentration, the three-layer density gradient is the best choice for these purposes. In addition, aneuploidy frequencies are lower in specimens enriched by the gradient centrifugation compared with unprocessed semen. A study shows that semen processed by density gradient centrifugation is very efficient in reducing sperms with aneuploidy and diploidy. ${ }^{11}$

The two-layer density gradient sperm preparations have a greater total sperm concentration than the three-layer gradient sperm preparations. It was concluded that the selected method should depend on the proposed clinical use. If the sperm preparation is performed for IUI, it is better to use the twolayer density gradient method to reach a higher total sperm concentration. If the goal is that the sperms are to be used for IVF or ICSI, it is better to use the three-layer density gradient method.

\section{References}

1. Al-Hasani $S$, AlpÜStÜN $S$, Ludwig $M$, Diedrich $K$, Bauer $O$, KÜPker $W$, et al. The combination of two semen preparation techniques (glass wool filtration and swim-up) and their effect on the morphology of recovered spermatozoa and outcome of IVF-ET. Int J Androl. 1996;19(1):5560.
2. Chen MJ, Bongso A. Comparative evaluation of two density gradient preparations for sperm separation for medically assisted conception. Hum Reprod. 1999;14(3):759-64.

3. Hammoud AO, Gibson M, Peterson MC, Carrell DT. Effect of sperm preparation techniques by density gradient on intra-individual variation of 
sperm motility. Arch Androl. 2007;53(6):349-51.

4. Centola GM, Herko R, Andolina E, Weisensel S. Comparison of sperm separation methods: effect on recovery, motility, motion parameters, and hyperactivation. Fertil Steril. 1998;70(6):1173-5.

5. Sakkas D, Manicardi GC, Tomlinson M, Mandrioli $M$, Bizzaro D, Bianchi PG, et al. The use of two density gradient centrifugation techniques and the swim-up method to separate spermatozoa with chromatin and nuclear DNA anomalies. Hum Reprod. 2000;15(5):1112-6.

6. Sbracia M. Sperm function and choice of preparation media: comparison of percoll and accudenz discontinous density gradients. J Androl. 1996;17(1):61-7.

7. Natali I. Sperm preparation techniques for artificial insemination - comparison of sperm washing, swim up, and density gradient centrifugation methods. In: Manafi $M$, editor. Artificial insemination in farm animals. Iran: In
Tech; 2011. p. 115-22.

8. Amiri I, Ghorbani M, Heshmati S. Comparison of the DNA fragmentation and the sperm parameters after processing by the density gradient and the swim up methods. J Clin Diagn Res. 2012;6(9):1451-3.

9. Allamaneni SS, Agarwal A, Rama S, Ranganathan P, Sharma RK. Comparative study on density gradients and swim-up preparation techniques utilizing neat and cryopreserved spermatozoa. Asian J Androl. 2005;7:86-92.

10. Tsai YC, Lin MY, Chen SH, Kang CY, Tsai YT, Lin $\mathrm{LY}$, et al. Comparing the clinical outcomes of intrauterine insemination by two different density gradient preparation methods. J Chin Med Assoc. 2004;67(4):168-71.

11. Brahem S, Letaief K, Ben Ali H, Saad A, Mehdi M. Efficacy of the density gradient centrifugation method in eliminating sperm with aneuploidy. Andrologia. 2013;45(3):158-62. 